\title{
Magnesium impurities decide the structure of calcium carbonate hemihydrate
}

\author{
JULIE AUFORT ${ }^{1}$ AND RAFFAELLA DEMICHELIS ${ }^{2}$ \\ ${ }^{1}$ Curtin University \\ ${ }^{2}$ The Institute for Geoscience Research (TIGeR) \\ Presenting Author: julie.aufort@curtin.edu.au
}

Calcium carbonate is an ubiquitous mineral, found not only in geological settings, but also in living organisms as the main inorganic component of seashells, pearls and eggshells. As a major carbon reservoir, it also plays a role in the global carbon cycle and is often used for palaeoenvironmental and plaaeoclimate reconstructions. The anhydrous polymorphs of calcium carbonate are often found to be the final products of a series of extensive structural reorganisation and dehydration events involving the formation of amorphous calcium carbonate (ACC) particles and of hydrated calcium carbonate intermediates. Determining the structural, thermodynamic and vibrational properties of such phases thus plays a key part in deciphering the still debated pathways by which calcium carbonate nucleates from solution.

Recently, an experimental study reported the discovery of a new hydromorph of calcium carbonate, calcium carbonate hemihydrate $\mathrm{CaCO}_{3} \cdot 1 / 2 \quad \mathrm{H}_{2} \mathrm{O}$ [1]. We have analysed this new phase using both first-principles and classical theoretical methods [2]. Geometry optimization of the pure phase resulted in a structure with higher symmetry than that proposed from the experiment ( $P b c n$, space group number 60). The introduction of magnesium impurities in the hemihydrate structure was found to cause a distortion in the unit cell, which assumes the monoclinic arrangement $\left(P{ }_{l} / c\right)$ originally proposed. Vibrational properties and relative thermodynamic stabilitites of the pure and $\mathrm{Mg}$ substituted hemihydrate phases were also investigated. Based on the theoretical results, we suggest that the calcium carbonate hemihydrate compound contained a small fraction of $\mathrm{Mg}$ impurities in its structure, which is consistent with the Mg-rich experimental conditions.

[1] Zou Z. et al., Science 2019, 363, 396-400. [2] Aufort J., Demichelis R., Cryst. Growth Des. 2020, 20, 8028-8038. 